\title{
A single-session of mindfulness meditation expedites immediate motor memory consolidation to improve wakeful offline learning
}

\author{
James O. Brown ${ }^{\mathrm{a}}$, Alex Chatburn ${ }^{\mathrm{b}}$, David L. Wright ${ }^{\mathrm{c}}$, and Maarten A. Immink ${ }^{\mathrm{d} \bowtie}$ \\ ${ }^{a}$ Alliance for Research in Exercise, Nutrition and Activity, University of South Australia, Adelaide, South Australia, Australia \\ ${ }^{\mathrm{b}}$ Cognitive and Systems Neuroscience Research Hub, University of South Australia, Adelaide, Australia \\ 'Department of Health \& Kinesiology, Texas A\&M University, College Station, Texas, United States \\ ${ }^{\mathrm{d} S p o r t, ~ H e a l t h, ~ P h y s i c a l ~ A c t i v i t y ~ a n d ~ E x e r c i s e ~ R e s e a r c h ~ C e n t r e, ~ F l i n d e r s ~ U n i v e r s i t y, ~ A d e l a i d e, ~ S o u t h ~ A u s t r a l i a, ~ A u s t r a l i a ~}$
}

\begin{abstract}
Post-training meditation has been shown to promote wakeful motor memory stabilization in experienced meditators. We investigated the effect of single-session mindfulness meditation on wakeful and sleep-dependent forms of implicit motor memory consolidation in mediation naïve adults. Immediately after implicit sequence training, participants $\left(N=20,8\right.$ females, $M_{\text {age }}=$ 23.9 years \pm 3.3 ) completed either a 10-minute focused attention meditation $(\mathrm{N}=10)$, aiming to direct and sustain attention to breathing, or a control listening task. They were then exposed to interference through novel sequence training. Trained sequence performance was tested following a 5-hour wakeful period and again after a 15-hour period, which included sleep. Bayesian inference was applied to group comparison of mean reaction time (MRT) changes across training, interference, wakeful and postsleep time points. Relative to control conditions, post-training meditation reduced novel sequence interference $\left(B_{10}=6.61\right)$ and improved wakeful motor memory consolidation $\left(\mathrm{BF}_{10}=\right.$ 8.34). No group differences in sleep consolidation were evident $\left(\mathrm{BF}_{10}=\mathbf{0 . 3 8}\right)$. These findings illustrate that post-training mindfulness meditation expedites wakeful offline learning of an implicit motor sequence in meditation naïve adults. Interleaving mindfulness meditation between acquisition of a target motor sequence and exposure to an interfering motor sequence reduced proactive and retroactive inference. Post-training mindfulness meditation did not enhance nor inhibit sleep-dependent offline learning of a target implicit motor sequence. Previous meditation training is not required to obtain wakeful consolidation gains from post-training mindfulness meditation.
\end{abstract}

motor sequence learning; mindfulness meditation; attention; motor memory consolidation; implicit learning; serial reaction time task; Bayesian inference

Correspondence: maarten.immink@flinders.edu.au

\section{Introduction}

Training, often referred to as on-line learning is commonly accepted as being essential for motor skill acquisition. However, skill acquisition is not strictly limited to the actual time spent training. Rather, following training, offline learning processes continue to contribute to motor skill learning (Robertson, Pascual-Leone, \& Miall, 2004). These offline learning processes are assumed to include motor memory consolidation.

Motor memory consolidation has been described as involving both offline memory stabilization and enhancement (Immink, 2016; Korman et al., 2007). Memory stabilization is associated with reduced susceptibility of newly formed mo- tor memory to subsequent interference (Robertson, PascualLeone \& Miall, 2004; Walker \& Stickgold, 2004). Stabilization processes are thought to be critical in the first 6 hours after training to ensure newly formed motor memories are not degraded or interfered with by performance of other motor tasks (Shadmehr \& Brashers-Krug, 1997; Walker et al., 2003). Stabilization is exemplified by long-term maintenance of performance at equivalent levels observed at the end of training (Walker, 2005). In contrast to stabilization, offline enhancement is characterized by performance improvement relative to the end of training following a period without further training (Walker \& Stickgold, 2004).

A framework for motor memory consolidation has been offered that suggests memory stabilization can emerge during wakeful non-practice periods in the hours that follow training, whereas performance enhancement from consolidation relies on exposure to a period of sleep following training (Diekelmann \& Born, 2007; Walker, 2005). More recent findings propose that a night of sleep is not required to promote memory consolidation (Brokaw et al., 2016; Mednick et al., 2003; Nishida \& Walker, 2007). Instead, napping (Mednick et al., 2003; Nishida \& Walker, 2007) as well as some wakeful activities such as aerobic exercise (Rhee et al., 2016; Roig et al., 2012) and cognitive tasks (Brown \& Robertson, 2007) have been shown to support offline improvements. Importantly however, the emergence of wakeful and sleep related consolidation is not only influenced by activities undertaken following training but also by the degree of awareness of task features within the practiced motor skill (King et al., 2017); that is, whether the learned motor skill is of explicit or implicit nature. Knowledge of the learned nature of the task or explicit learning is thought to provide for sequence learning enhancements when a period of sleep is involved (Debas et al., 2010; Fischer et al., 2002; Walker et al., 2002), compared to a period of wakefulness showing no enhancement. And contrastingly, under implicit learning conditions (i.e. with no awareness of the learning requirement involved in the task) off-line enhancements have emerged only over wakefulness (Robertson, Pascual-Leone, \& Press, 2004).

It is not yet fully understood how post-training wakeful interventions may support motor memory consolidation. However, a unifying theme that has emerged across the demonstrations involving post-training interventions such as exercise, cognitive task performance and mindfulness meditation 
is attention control, and more specifically, these tasks require a heightened level of attention control to perform. Motor learning is known to rely on attentional resources (Dayan \& Cohen, 2011) and since goal-oriented tasks involve cognitive control processes needed to regulate attention (Chan et al., 2017), the addition of a goal-orientated task post-training might benefit motor memory consolidation through mechanisms of attention control.

For example, Brown and Robertson (2007) demonstrated that performing a word recall task following motor skill training provided performance enhancement when the skill was tested following a wakeful period. Word recall task performance has been associated with increased attention demands (Hertel \& Rude, 1991) and more broadly, cognitive tasks are commonly accepted as requiring attention control. Inducement of motor memory consolidation through post-training aerobic exercise (Rhee et al., 2016; Roig et al., 2012) may also be related to eliciting attentional control. Rhee et al. (2016), for example, described the exercise task as being perceived by the performer as another task that requires attention. More broadly, there is agreement that acute bouts of exercise elicit increased executive function including attention (Chang et al., 2012; Colcombe \& Kramer, 2003; Etnier et al., 1997; Lambourne \& Tomporowski, 2010; Tomporowski, 2003). Exercise might rely on increased attention allocation as part of maintaining exercise performance goals (e.g., pace, intensity level) which might require monitoring interoceptive information (McAuley et al., 2011; Schücker et al., 2013). The importance of attention in exercise is clearly demonstrated by impaired exercise performance when attention resources have been depleted by a preceding cognitive task (Marcora et al., 2009).

One additional post-training wakeful intervention that has recently been shown to support motor memory consolidation, mindfulness meditation, appears to more directly implicate attention control as important for offline gain (Immink, 2016). Rather than relaxation, meditation has been described as a goal-oriented cognitive task (Chan et al., 2018; Immink et al., 2017). Importantly, meditation explicitly targets the manipulation of attention, since the goal of meditation is to direct and sustain attention to an object or experience such as body sensations or breathing (Malinowski, 2013; Tang et al., 2015), and thus meditation can be considered a form of attentional cognitive training. To achieve this goal, meditation techniques rely on engagement of attention control processes (Chan et al., 2020; Chan et al., 2017; Chan et al., 2018; Immink, 2016; Immink et al., 2017; Malinowski, 2013; Tang et al., 2015). As cognitive tasks, bouts of exercise and mindfulness meditation can be conceived as goal-oriented tasks, and these post-training interventions might have a shared capacity to support wakeful motor memory consolidation through eliciting increased attention control (Colzato et al., 2013; Colzato et al., 2017; De Bruin et al., 2016).

Like exercise and cognitive task performance interventions, attention control might be a phenomenon that is able to explain meditation as a potential regulator in motor memory consolidation. Attention is a central component of medi- tation practice, where the training of attention brings about the ability to maintain non-judgmental awareness of one's thoughts, feelings and experiences - a primary aim of meditation (Malinowski, 2013). Maclean et al. (2010) showed improved sustained attention in experienced meditators with intensive (5hr/day for 3 months) meditation practice. Others have shown that intensive practice with experienced meditators is not necessarily required to elicit improvements in attention control. Norris et al. (2018) show that a brief 10minute audio guided meditation improves attention allocation in novice meditators. This was established through increased event-related brain potential (ERP) N2 amplitudes which are implicated in attention control. Chan et al. (2020) had novice meditators complete 22-minute daily sessions of focused-attention meditation (FAM) over 21 days prior to a serial reaction time task (Nissen \& Bullemer, 1987). Participants who completed FAM demonstrated faster reaction time (RT) performance and augmented N2 amplitudes over anterior and central regions during the SRTT, indicating that FAM can foster changes in neuronal activity related to improved self-regulation of attentional control (Moore et al., 2012).

Performance gains from motor memory consolidation as a result of increased demand for attention control may be associated with recruitment of anterior cingulate cortex (ACC) and dorsolateral prefrontal cortex (DLPFC) during meditation (Cahn \& Polich, 2006). Using neuroimaging, Baerentsen et al. (2001) has shown mindfulness meditation to reflect state increases in both anterior cingulate cortex (ACC) and dorsolateral prefrontal cortex (DLPFC) activity. These effects have also been replicated in other studies using alternative methods of meditation (Lazar et al., 2000; Newberg et al., 2001). In the context of FAM, DLPFC activity is thought to be involved in repeatedly redirecting or sustaining attention to object focus (Lippelt et al., 2014); and similarly, the ACC is thought to be involved in the refocusing of attention. As Hasenkamp et al. (2012) highlighted, as the mind starts to wander during meditation, the ACC plays a role of 'error' detection and provide feedback to executive control networks (Lippelt et al., 2014). The DLPFC has also been implicated in the consolidation of procedural skills (Tunovic et al., 2014). By modifying corticospinal excitability with theta burst stimulation to the DLPFC, a consistent relationship was found between corticospinal excitability and offline improvements. Specifically, corticospinal changes differed in the context of implicit and explicit sequence learning tasks. For example, implicit motor sequences produced no change in corticospinal excitability and provided for subsequent offline improvements over wakefulness, whereas following the explicit motor sequence task, corticospinal excitability was reduced, allowing for no offline improvements. However, continuous stimulation applied to the DLPFC following the explicit task eliminated the decrease in corticospinal excitability and therefore induced substantial offline improvements (Tunovic et al., 2014).

Research investigating the role of meditation state effects on neural activity using electroencephalography (EEG) has revealed fluctuations in both alpha and theta band activity 
(Cahn \& Polich, 2006; Larson et al., 2013). Evidence of increased alpha power has been shown during FAM and open monitoring (OM) meditations (Lee et al., 2018) compared to controls (Cahn \& Polich, 2006). In particular, FAM is associated with a bilateral increase in alpha power, while OM reflecting a decrease only in left-sided power (Lee et al., 2018). The emergence of theta waves in frontal regions during meditation highlights involvement in attentional processes (Baijal \& Srinivasan, 2010), particularly, in areas such as the ACC, medial prefrontal cortex and DLPFC which are importantly linked with attention-demanding tasks (Cahn \& Polich, 2006).

Immink (2016) demonstrated that motor memory stabilization could be induced by meditation following training on an explicit motor sequence task. Twelve experienced meditators were trained on a sequential key pressing task in the morning and then completed a 30-minute session of FAM or light work duties (control group) at midday. At about 5:00 pm of the same day, participants completed testing on trained and novel sequences. At test, the meditation group performed trained sequences faster than the control group but there were not group differences in performance of novel sequences. This pattern highlighted that the advantage of post-training meditation was not due to general performance enhancement, through increased alertness at test, for example, but rather, was specific to previously practiced sequences. Moreover, the meditation group exhibited equivalent performance levels with the trained sequence between the final training block and the wakeful test, with the control group exhibiting performance losses between these timepoints. This demonstration of memory stabilization in the meditation group can be explained by an enhanced level of motor chunking afforded by meditation following the previously trained sequences. Motor chunking has been shown to play an important role in both explicit and implicit sequence learning (Dahms et al., 2020) and given the faster RT in the meditation group, this may have reflected enhanced response planning and execution processes offered by meditation.

The present experiment aims to build on findings from Immink (2016) firstly by investigating motor memory consolidation in meditation naïve individuals. Tang et al. (2015) have developed a framework describing the core components of meditation which suggests that experienced meditators are thought to effortlessly reduce mind-wandering or intermittent shifts away from the task at hand (Mrazek et al., 2012) through the practiced ability to monitor attention (Malinowski, 2013). Immink (2016) included only experienced meditators who reported a mean of 9 years of practice. This demonstration of memory stabilization is consistent with the framework in signifying that experienced meditators may not wind-wander, distinct from beginner meditators who are thought to be less adept at monitoring attention and therefore unable to readily detect when mind wandering is occurring (Malinowski, 2013). However, Chan et al. (2018) saw increased attention control associated with improved performance when novices completed meditation prior to participating in a sequence learning task. This suggests that be- ginners may too derive benefits for learning via meditation and highlights the need to revisit the work produced by Immink (2016) to explore beginner meditative states following training. Provided beginners can derive these learning improvements from a single session of meditation would provide a considerable increase in the number of individuals that can employ these benefits rather than exclusively experienced meditators. Therefore, it was hypothesized that meditation naïve participants can derive sequence learning benefits from a single session of meditation (Chan et al., 2018) in the form of memory stabilization (Immink, 2016).

Secondly, distinct from Immink (2016) where an explicit sequence task was used, the present experiment included an implicit sequence task. As previously mentioned, an explicit sequence task has exhibited subsequent offline improvements only over a period of sleep (Debas et al., 2010; Fischer et al., 2002; Walker et al., 2002), and not over wakefulness. The findings by Immink (2016) are in line with this notion since meditation in the wakeful period following training exhibited memory stabilization of the explicit task. In contrast, off-line enhancement using an implicit sequence task has emerged only over wakefulness (Robertson, Pascual-Leone, \& Press, 2004). Including an implicit sequence task in the present experiment allowed for the possibility of wakeful offline improvements following the insertion of meditation. It was hypothesized that a single session of meditation following training would allow for these improvements to occur despite the inclusion of an additional sequence designed to retroactively interfere with the acquisition of the initial implicit sequence.

Thirdly, the present experiment included a post-sleep test to investigate the influence of a post-training meditation intervention on sleep-dependent consolidation. This was beyond the scope of Immink (2016) which removed the possibility for post-meditation improvements which may require sleep to emerge in the context of an explicitly learned skill (Fischer et al., 2002; Stickgold et al., 2000; Walker et al., 2002). A synergistic effect might be observed when coupling post-training meditation and sleep-related consolidation when comparing offline improvements provided by sleep only. Research involving meditation practitioners and sleepdependent procedural consolidation attribute improvements in task performance to a lower density of occipital sleep spindles in meditators, whereas in non-meditators, offline gains were associated with an increased time spent in REM sleep. This suggests meditation practice might offer different sleepdependent consolidation patterns to those of non-meditators (Solomonova et al., 2020). Given what is known about sleep as a promoter for offline improvements in motor behaviours, it is evident that further research incorporating a meditation intervention to test motor memory consolidation following a period of sleep is required. It was hypothesized that post-training meditation would support sleep-dependent offline learning. Offline learning would be reflected through enhanced performance in the post-sleep test relative to endof-training and the wakeful period test.

Lastly, a single session of meditation was inserted immediately following training distinct from Immink (2016) where a 
delay was implemented between the end of training and meditation. The introduction of meditation 4 hours after training seemed to have encouraged motor memory stabilization as opposed to improvement (Immink, 2016). However, work by Rhee et al. (2016) has demonstrated stronger consolidation effects when participants were subjected to the post-training intervention immediately following training. It was hypothesized that implementing meditation immediately following motor sequence learning would promote motor memory consolidation. If so, it was assumed that post-training meditation would enhance motor memory stabilization across a wakeful period. Motor memory stabilization was established by performance on a wakeful period test being equivalent to that of end-of-training performance.

\section{Methods}

\section{Participants}

Twenty healthy, meditation naïve adults ( 2 left-handed, 1 mixed-handedness, 8 females, $M_{\text {age }}=23.9$ years \pm 3.3 ) agreed to participate in the present experiment. Participants were required to have no previous formal experience with both meditation practice and mental skills training. During participant recruitment and provision of instructions reference to mindfulness or meditation was purposefully avoided to prevent any expectations or biases related to these concepts (Davidson \& Kaszniak, 2015). The present experiment was approved by the University of South Australia Human Research Ethics Committee and all participants provided written informed consent prior to participation.

Serial Reaction Time Task The SRTT (Nissen \& Bullemer, 1987) is a four-choice, finger-tapping task used to study cognitive and biological aspects of learning and memory (Robertson, 2007) and more specifically, to measure observational motor learning of sequential information (Heyes \& Foster, 2002). The SRTT (see Figure 1A) involved the presentation of a row of four boxes on a computer screen that represented four response keys numbered 1-4 (from left to right) on a modified keyboard (Chan et al., 2018). Participants were instructed to place their index and middle fingers on keys 1 and 2 (left hand) and keys 3 and 4 (right hand). A response was required when a stimulus appeared at any one of the four positions (i.e. a box was filled red). Here, the participant selected the appropriate response by pressing the corresponding key as fast as possible. A trial consisted of the presentation of one stimulus and the production of one response. At the end of each trial, a 50ms fixed delay occurred before the presentation of a new response stimulus for the next trial. Feedback was provided for inaccurate key presses and a $250 \mathrm{~ms}$ too-long response delay, both displayed as an error tone. Performance feedback, that is, block averaged RT and number of error trials was provided at the end of each block. Following the presentation of performance feedback, participants received a 15 -second rest interval before the beginning of the next block (Chan et al., 2018).

In the SRTT, a single block was comprised of 120 trials where participants completed a total of 24 blocks in train- ing (session 1) and 3 blocks in both the wakeful period test (session 2) and the post-sleep test (session 3). Within a block, the stimulus was played out in the order of a specific implicit target sequence (target sequence, interference sequence, and recognition sequences). The target and interference sequences both contained 12-items, whereas the recognition sequences consisted of 4-items each. Within training, participants completed 12 blocks of the target sequence (e.g. 1-2-1-3-4-2-3-1-4-3-2-4) (Reed \& Johnson, 1994) and subsequently 12 blocks of the interference sequence (e.g. 1-2-4-31-3-2-1-4-2-3-4) (Dumel et al., 2016) to introduce retroactive interference on the target sequence. A measure of sequence learning was acquired by comparing the reaction times of the initial target sequence and those of the interference sequence (Robertson, 2007). The target sequence was re-introduced for both the wakeful period and post-sleep tests, with the latter test including additional recognition target sequences comprising of elements from the target and interference sequences (e.g. 1-2-1-3-4; 4-3-2-4 etc.). The recognition test involved 36 trials of a given sequence displayed on the screen where participants were required to correctly recall the sequence using the same finger-placement as the target and interference sequences. After successfully pressing each 4item sequence, a response of either "yes, this sequence was part of the previous sequence" or "no, this sequence was not part of the previous sequence" was required. All sequences were offered in a pseudorandomized manner with each response stimuli not repeated consecutively and equally represented within each block. The SRTT was presented using E-Prime 3.0 Software (Psychology Software Tools Inc., Pittsburgh, PA) on a 58.4cm-wide Alienware AW2310 LCD display running at $1680 \times 1050$ pixel resolution in 64-bit colour, with a screen refresh setting of $120 \mathrm{~Hz}$.

Mindfulness Meditation and Control Participants were randomly allocated to either a Meditation or Control group. Participants assigned to the Meditation group completed a single session of meditation following the completion of training on the target sequence. The 10-minute meditation technique was based on a meditation technique previously used before motor sequence training to enhance sequence learning in meditation naïve participants (Chan et al., 2020; Chan et al., 2017; Chan et al., 2018; Immink et al., 2017); and enhancement of motor memory consolidation with experienced meditators (Immink, 2016). The meditation technique, which was completed with the eyes closed while seated on a chair in a distraction free room, instructed participants to direct their attention to their breathing. Along with aiming to direct and sustain attention to breathing, participants were instructed to monitor for instances where their attention was distracted from breathing. In the event of distraction, participants were instructed to 'let go' of the distraction and redirect attention to breathing. Breath focusing techniques such as this have been validated as a reliable behavioural measure of mindfulness as it has been related to a reduced level of mind-wandering (Levinson et al., 2014). This method can be thought of as suitable for beginner meditators as breath counting requires little effort and arousal compared to other 


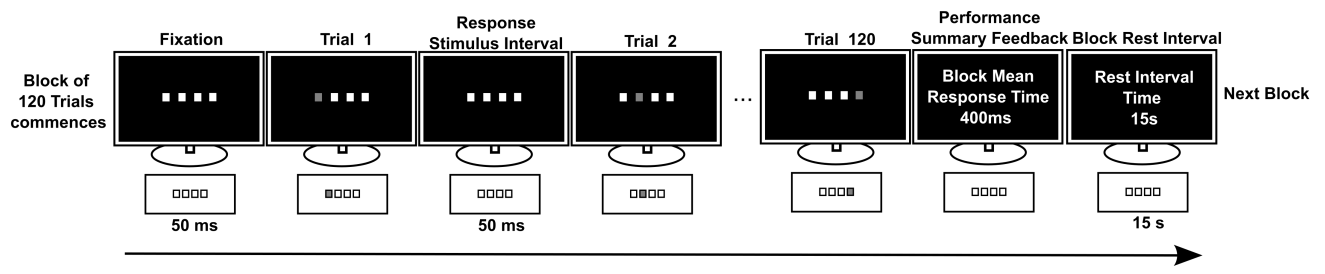

B

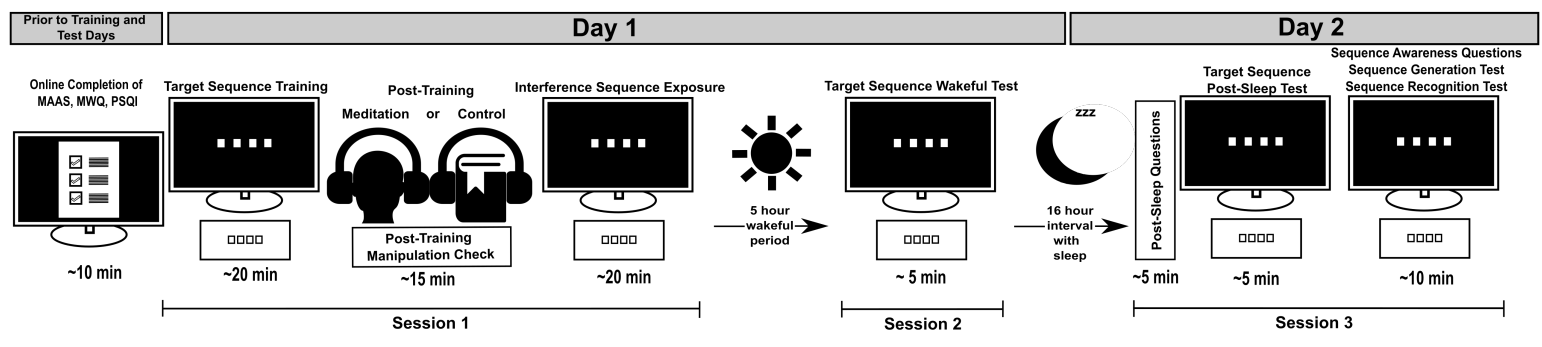

Fig. 1. Example serial reaction time task (SRTT) trial and Experiment procedure. (A) SRTT stimuli were spatial mapped to key press responses, performed by the middle and index fingers of the left and right hand and numbered 1 to 4 from left to right. Unbeknownst to participants, stimulus presentation followed a 12 -item second-order conditional sequence in training (1-2-1-3-4-2-3-1-4-3-2-4, Reed \& Johnson, 1994) and interference (1-2-4-3-1-3-2-1-4-2-3-4, Dumel et al., 2016) phases. (B) The procedure involved an $\mathrm{AB}$ paradigm for target sequence training followed by exposure to an interference sequence. Participants experienced a single session of mindfulness meditation or audiobook listening, as control condition, prior to exposure with the interference sequence. Wakeful and sleep-dependent forms of motor memory consolidation were examined with a same day wakeful test and 24-hour delayed post-sleep test, respectively. The extent of explicit versus implicit sequence was assessed with follow-up awareness questions, a sequence generation task and then a sequence recognition task.

meditation practices (Lumma et al., 2015). Participants allocated to the Control group listened to a 10-minute audio recorded reading excerpt from the text Your Mind and How to Use It (Atkinson, 1911) following the completion of training on the target sequence. As within the Meditation group, the control group task was completed with eyes closed, while seated in a chair in a distraction free room.

Procedure The experimental procedure is illustrated in Figure 1B. Eligible participants were sent a link to complete a 10-minute online questionnaire using an online survey platform (LimeSurvey, LimeSurvey Project Team, Hamburg, Germany). Initially participants provided written informed consent and demographic details such as age, gender, handedness, and the highest level of education completed. These details were collected to potentially explain baseline differences in task performance.

Participants completed the Mind-Wandering Questionnaire (MWQ) developed by Mrazek et al. (2013) as a measure of individual trait levels of task-unrelated thought. A reliability analysis of this tool has revealed a Cronbach's alpha of 0.850 which indicates good consistency between questions (Mrazek et al., 2013). The MWQ investigates mind-wandering qualities to provide nuanced descriptions of an individual's mental state (Davidson \& Kaszniak, 2015; Shiffman et al., 2008). Here it was used to identify potential participant characteristics related to their capacity to complete the Meditation or Control groups. Thereafter, participants completed the Mindful Attention Awareness Scale (MAAS) adapted from Brown and Ryan (2003). This 15-item scale was designed to assess the central feature of mindfulness, that is, attention (Malinowski, 2013; Tang et al., 2015); by using a rating scale to indicate the frequency of a given statement about one's everyday experience (Brown \& Ryan, 2003). Here, participants were required to respond on a 6-point Likert scale from 1 (always) to 6 (almost never) to the given statements where a higher mean score indicating a higher level of mindfulness awareness. MAAS scores are collected as potential mediators of meditation and control effects on performance and/or consolidation. The MAAS has been demonstrated as a valid measure of trait mindfulness (Black et al., 2012; Brown et al., 2011; Carlson \& Brown, 2005; Osman et al., 2016) with a Cronbach's alpha of 0.81 . Notable research by Levinson et al. 
(2014) demonstrated that breath counting accuracy was positively linked with self-reported trait mindfulness using the MAAS thereby applying the validity to a meditation setting. Finally, participants completed the Pittsburgh Sleep Quality Index (PSQI) developed by Buysse et al. (1989) as a selfrated questionnaire that aims to assess sleep quality and disturbances over a one-month time frame (Buysse et al., 1989). The PSQI is a 19-item scale grouped into seven components with each yielding a score from 0 to 3 . These sleep component scores are grouped to provide a global score between 0 to 21, with a higher global score indicating poorer sleep quality (Zhong et al., 2015). The PSQI has been demonstrated as a valid measure for sleep quality, correctly identifying $88.5 \%$ of good and poor sleepers and reporting a Cronbach's alpha of 0.83 in the original work (Buysse et al., 1989). PSQI results were collected as a baseline measure for potential individual differences to assess whether sleep quality has an impact on motor memory consolidation.

Following completion of the online questionnaire, participants were invited to complete three experimental sessions over two consecutive days. Seventeen participants completed all three sessions in a laboratory while three participants completed their sessions remotely due to health restrictions associated with the COVID pandemic. Remote completion of the sessions was based on sending participants download links via email to access E-Prime Go (Psychology Software Tools Inc., Pittsburgh, PA) executable files that ran the experiment scripts used in the laboratory. During remote sessions, an experimenter was present via a Zoom video call to answer any questions the participants may have had about the task, and ensure the sessions were completed appropriately under laboratory-like conditions (i.e. little distraction from noise, no use of devices while completing the task etc.). The remote experiment software required participants to use the Windows operating system. Remote participants completed the SRTT using a standard QWERTY keyboard, producing responses with the $\mathrm{V}, \mathrm{B}, \mathrm{M}, \mathrm{N}$ keys. Once a session was completed, participants sent the acquired data file back to the experimenter via email. Participants then deleted the session script and data file from their computer.

Session one, which commenced between 8:00 and 10:00 am, first involved target sequence training in the SRTT over 12 blocks, each consisting of 120 trials or 10 cycles of the 12item sequence. Between blocks, participants were first provided with summary feedback of block mean accuracy and reaction time followed by a 30-second rest break. In the posttraining period immediately following target sequence training, participants completed 10 minutes of either the Meditation or Control audiobook. For both, the audio recording ran automatically after SRTT training within the E-Prime script. Immediately after completion of post-training meditation or audiobook listening, participants completed a series of questions related to their experience with the posttraining task. The questions were delivered within the Eprime script. Participants first answered an open-ended question asking them to describe what they were doing in the previous post-training task. Responses were typed into a text response box. Participant responses were evaluated as a manipulation check that the Meditation group was directing attention to the breath as instructed and the Control group was listening to the audiobook and importantly, no directing attention to the breath. Next, participants used a computer mouse (or track pad for remote participants) to rate their posttraining experience along a 100-point visual analogue scale (VAS) similar to Immink et al. (2017). The VAS scale was about a $10 \mathrm{~cm}$ horizontal line with text anchors on the left (labelled 'None') and right (labelled 'Maximum'). Participants completed separate VAS scales for the following ratings: 1) "How often did your mind wander while completing the previous task?" (Distraction), 2) "How demanding was the previous task for you?" (Effort), 3) "How motivated were you to perform the previous task?" (Motivation). These subjective ratings were included to provide some account of the participant experience in the post-training conditions (Davidson \& Kaszniak, 2015). Finally, for the first session, immediately after completion of post-training condition ratings, participants completed 12 blocks of the SRTT with the interference sequence. Exposure to this sequence was intended to compare the degree of proactive interference between the target and interference sequences between Meditation and Control groups. Importantly, to consider the stabilization form of motor memory consolidation, we also compared the extent of retroactive interference from the interference sequence on the target sequence (Song, 2009) between Meditation and Control groups. In the latter case, retroactive interference would be exhibited at a wakeful test as diminished target sequence performance relative to the end of training.

About 5-hours after completion of session one, participants completed the second session that included a wakeful test of performance on the target sequence. Then, about 24-hours after the completion session of 1 , a period which included a bout of sleep, participants completed the third session which first asked participants to report their time in bed the prior night and the time out of bed in the present morning. These times were used to calculated time in bed. Next, participants rated their overall quality of their prior sleep on a 100-point VAS. Participants then completed a post-sleep test of the target sequence. The wakeful and post-sleep tests each included 3 blocks of the SRTT conducted with similar conditions to training in terms of trials per block, provision of summary feedback and inter-block rest intervals. Following completion of the post-sleep test, participants completed a series of questions aimed to assess their awareness of the presence of a sequence in the SRTT (Robertson, 2007). First, participants were asked to type in their response to the open-ended question, "Describe what strategies you used to perform the key pressing task." These responses were inspected to determine if the participant had awareness of the embedded sequence based on responses which included the keywords, "pattern", "order" or "sequence." Participants were then asked to indicate if the order of the response stimuli followed a pattern or was random. Third, participants were then informed that the order of stimuli followed a sequence pattern and were then asked to rate their confidence in being able to repro- 
duce the sequence on a 100-point VAS scale. Text anchors were labelled as follows: Left anchor 'None', between Left and Middle 'Some of the Pattern', Middle anchor 'Half of the Pattern', Between Middle and Right 'Most of the Pattern, and Right anchor 'The Entire Pattern'. Participants were then asked to complete a recall test of the 12-item sequence followed by a recognition test. For the recognition test, the trained sequence was decomposed into 12 4-item sequence chunks, where each chunk commenced by each sequence position (for sequence positions 10, 11 and 12, the sequence included the initial sequence elements). Another 12 4-item chunks were similarly formed based on the interference sequence and lastly 124 -item chunks that were unique to those from trained and interference sequences. The 36 chunks were presented individually in a random order across the 36-trial recognition test. Participants were not informed if the sequence was based on the trained or novel sequence. Each trial involved the same stimulus and response format as the SRTT except the sequence only involved four stimuli and key presses. After completing the 4-item sequence, the participant had to indicate if that sequence was part of the sequence they previously performed with a yes or no response.

Data Analysis

Meditation and Control group comparisons of participant characteristics, training, wakeful and post-sleep SRTT performance and sequence awareness, recall and recognitions were undertaken using Bayesian inference. Bayesian analysis of the data were conducted in JASP ver. 0.14.1 (JASP Team 2020) Bayesian inference offer several advantages over null hypothesis statistical tests for data inferences. Bayes Factors (BF) are useful when determining the presence or absence of an effect particularly in small sample sizes (Makowski et al., 2019; Van Doorn et al., 2020).

To test that random allocation did not result in group differences in participant characteristics, Bayesian chi-square test was used to test for equivalent gender and handedness distribution, while 2-tailed Bayesian independent samples ttest was used to infer if groups differed with respect to age, mind wandering (MWQ), mindfulness disposition (MAAS) and history of sleep quality (PSQI) as well as time in bed and quality of sleep VAS score prior to session 3 post-sleep performance test.

For SRTT performance data, trials with inaccurate responses were first removed. This resulted in removal of $2.3 \%$ of the data, which is below the acceptable exclusion rate of $5 \%$ for SRTT studies (Chan et al., 2018). Next, for each participant, MRT was calculated for each block of training, interference, wakeful and post-sleep testing sessions. Group-level MRT and standard deviation were then calculated and plotted to visualise the direction of any potential group differences, see Figure 2A. This visualisation allowed for a priori specification of one-tailed Bayesian independent samples ttest. Visual inspection was also conducted to evaluate if data met assumptions of normality and equal variance. This was followed up with Shapiro-Wilks' test of normality and Levene's test of variance homogeneity. As some of the data did not meet tests of normality and homogeneity assumptions, non-parametric versions of the Bayesian t-tests indicated below were conducted based on Bayesian Mann-Whitney U Test Bayesian for independent-samples t-tests and Wilcoxon Signed-Rank Test for single-sample t-tests. Both tests involved five chains of 10,000 iterations.

As MRT was shorter for the Control group, a one-tailed Bayesian independent t-test was used to test the alternate hypothesis that MRT was less for the Control group than the Meditation group at training block 1 (early training) and block 12 (training end). In addition, a two-tailed Bayesian independent sample t-test was conducted to test for group differences in training improvement, see Figure 2B. Training improvement was calculated for each participant as the difference in MRT between training block 1 and 12 .

As a manipulation check, we evaluated the frequency of reported attention focusing versus listening keywords from participant written post-training Meditation and Control task reports with Bayesian chi-square. Next, we conducted three separate two-tailed Bayesian independent samples t-test to test the alternate hypothesis that groups differed in VAS scores for ratings of distraction, effort and motivation in posttraining tasks.

Proactive interference of the training sequence on the interference sequence was compared between participants exposed to post-training meditation or the control audiobook. For this, the difference in MRT between training block 12 and interference block 1 was calculated for each participant, with higher values reflecting increased slowing due to proactive interference. As interference slowing was descriptively higher for the Control group, these change values were submitted to one-tailed Bayesian independent samples t-test to test the alternative hypothesis that MRT slowing was higher in the Control group, see Figure 2C.

Wakeful and sleep-dependent forms of motor memory consolidation were evaluated by calculating for each participant MRT changes between training block 12 and wakeful test block 1 and wakeful test block 3 and post-sleep block 1 . In these change values, larger negative values reflect increasing MRT improvement, while larger positive values reflect increased MRT performance loss and zero value reflects no change in performance. As these change values were descriptively larger for the Control group, they were separately analysed with one-tail Bayesian independent samples t-tests to test the alternate hypothesis that the Control group had higher MRT differences, see Figure 2D, E. Additional, Bayesian single-sample t-tests were conducted to test if MRT differences were not equal to zero in each group.

Using Bayesian chi-square inference, data from written responses to performance strategy were analysed for group differences in the frequency of reported keywords reflecting awareness of a pattern or sequence and separately, we evaluated if groups differed with respect to frequency of reporting if the order of stimulus presentation was random or followed a pattern. Group differences in sequence reproduction confidence ratings and recall accuracy percentage were evaluated with separate two-tailed Bayesian independent samples t-tests. Recall accuracy percentage was calcu- 

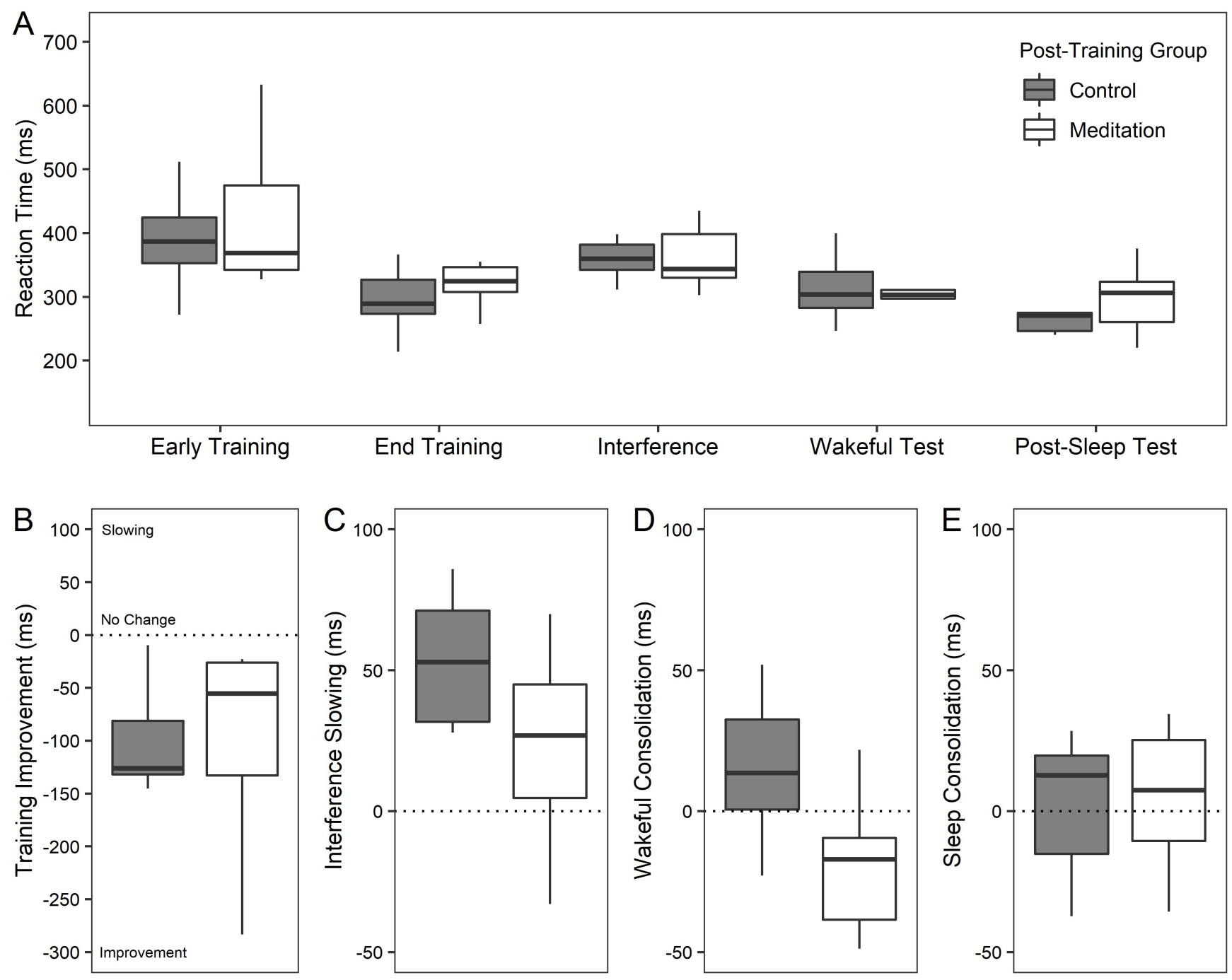

Fig. 2. Mean RT performance and performance changes at training, interference, wakeful test and post-sleep test phases. (A) Boxplot of mean RT comparison between Meditation and Control post-training groups for the first (early training) and last (end training) training blocks, the first interference sequence block, first wakeful test block and first post-sleep test block. Participants completed either 10 minutes of audio book listening (Control) or audio-guided mindfulness meditation (Meditation) immediately after completion of trained sequence training. (B) Training improvement was based on reaction time improvement between the first and last training block. (C) Interference slowing based on reaction time slowing between the final block of training on the target sequence and the introduction of the interference sequence. (D) Wakeful consolidation was based on reaction time change between the final training block and the first wakeful test block. The wakeful test was performed approximately five hours after completion of target sequence training, with post-training Meditation or Control post-training conditions and then exposure to an interference sequence intervening training and wakeful test. (E) Sleep consolidation was based on reaction time change between the wakeful test and post-sleep test. The post-sleep test was completed approximately 15 hours after the completion of the wakeful test and this interval included a normal night's sleep.

lated for each participant based on the number of key presses that matched the trained sequence. Single-sample Bayesian t-test was conducted separately for Meditation and Control groups to test if recall percentage was above the $25 \%$ guess rate. Sequence recognition test performance was evaluated based on the percentage of hits for target sequence chunks, percentage of hits for interference sequence chunks, and percentage of correct rejections for novel chunks. Group differences in these percentages were evaluated with three separate two-tailed Bayesian independent samples t-tests. This analysis was followed up with single-sample Bayesian t-tests to evaluate if hit and correct rejection rates were above the 50\% guessing rate in Meditation and Control groups.

For all Bayesian inferences, uninformed Cauchy distributions of $r=0.707$ with an effect size of 0 were used for prior probabilities since little prior knowledge exists from previous work applying Bayesian inference in the present context (Van Doorn et al., 2020). For non-parametric Bayesian ttests, inferences in favour of the null or alternate hypothesis were based on a Bayes Factor, $\mathrm{BF}_{10}$ which indicates the degree of evidence in support of the alternative hypothesis $\left(\mathrm{H}_{1}\right)$ versus the degree of evidence in support of the null hypothesis $\left(\mathrm{H}_{0}\right)$. While the Bayes Factor represents a continuum of evidence, to describe the level of evidence we applied heuristic labels described by Van Doorn et al. (2020) for ranges of $\mathrm{BF}_{10}$. We report the median effect size ( $\delta$ ) and $95 \%$ credible interval for the effects size $(95 \% \mathrm{CI})$ for the from Bayesian independent t-test evaluation of group data. Finally, for ease of communicating the effect of post-training meditation on motor memory consolidation outcomes, we calculated effect 
Table 1. Participant gender, handedness, age characteristics, and Mindfulness Attention Awareness Scale (MAAS), Mind Wandering Questionnaire (MWQ), and Pittsburgh Sleep-Quality Index (PSQI) scores for by post-training Control and Meditation groups.

\begin{tabular}{|c|c|c|c|c|c|c|c|c|c|c|c|}
\hline & \multicolumn{2}{|c|}{ Participant Count } & \multirow[b]{3}{*}{ Mean (SD) } & \multicolumn{2}{|c|}{ Age (yrs) } & \multicolumn{2}{|c|}{$\begin{array}{l}\text { Mindful Attention Awareness } \\
\text { Scale }\end{array}$} & \multicolumn{2}{|c|}{ Mind Wandering Questionnaire } & \multicolumn{2}{|c|}{ Pittsburgh Sleep Quality Index } \\
\hline & Control & Meditation & & Control & Meditation & Control & Meditation & Control & $\begin{array}{l}\text { Meditation } \\
\end{array}$ & Control & Meditation \\
\hline $\mathbf{N}$ & 10 & 10 & & $23.3(1.70)$ & $23.3(2.54)$ & $3.89(0.82)$ & $4.42(0.82)$ & $3.16(0.63)$ & $2.89(1.14)$ & $5.90(1.97)$ & $3.70(2.16)$ \\
\hline & \multicolumn{2}{|c|}{ Gender Distribution } & $95 \% \mathrm{Cl}$ & \multicolumn{2}{|c|}{$[22.08,24.52][21.48,25.12]$} & {$[3.31,4.48]$} & {$[3.84,5.00]$} & {$[2.70,3.61]$} & {$[2.06,3.70]$} & {$[4.49,7.31]$} & {$[2.15,5.25]$} \\
\hline $\mathrm{N}$ Females & 4 & 4 & Alternate hypothesis & \multirow{2}{*}{\multicolumn{2}{|c|}{0.40}} & \multicolumn{2}{|c|}{ Control ₹Meditation } & \multicolumn{2}{|c|}{ Control = Meditation } & \multicolumn{2}{|c|}{ Control ₹Meditation } \\
\hline $\mathrm{BF}_{10}$ & \multicolumn{2}{|c|}{0.50} & $\mathrm{BF}_{10}$ & & & \multicolumn{2}{|c|}{0.81} & \multicolumn{2}{|c|}{0.47} & \multicolumn{2}{|c|}{2.47} \\
\hline & \multicolumn{2}{|c|}{$\begin{array}{c}\text { Handedness } \\
\text { (L), (R), (A) }\end{array}$} & & & & & & & & & \\
\hline & $(0),(10),(0)$ & (2), (7), (1) & & & & & & & & & \\
\hline $\mathrm{BF}_{10}$ & \multicolumn{2}{|c|}{0.50} & & & & & & & & & \\
\hline
\end{tabular}

sizes for all group comparisons of SRTT performance based on Cohen's d.

\section{Results}

Participant Characteristics

There was no evidence for group differences in gender distribution $\left(\mathrm{BF}_{10}=0.50\right)$, handedness $\left(\mathrm{BF}_{10}=0.504\right)$, age $\left(\mathrm{BF}_{10}\right.$ $=0.40$, error $\%=7.0^{\mathrm{e}-6}$ ), or mindfulness disposition (MAAS scores, $\mathrm{BF}_{10}=0.81$, error $\%=2.2^{-4}$ ). There was only anecdotal evidence for group differences in background sleep quality (PSQI scores, $\mathrm{BF}_{10}=2.47$, error $\%=4.4^{\mathrm{e}-4}$ ). There was also anecdotal evidence for differences in overall MRT across all phases for participants who completed the experiment either in the laboratory or remotely (Training, $\mathrm{BF}_{10}=0.55$, error $\%=0.001 ;$ Interference, $\mathrm{BF}_{10}=0.75$, error $\%=0.004$; Wakeful Test, $\mathrm{BF}_{10}=0.56$, error $\%=0.001$; Post-Sleep Test, $\mathrm{BF}_{10}=0.49$, error $\left.\%=8.646^{\mathrm{e}-6}\right)$. Summary of participant characteristics by group are presented in Table 1 .

Mean RT at training and proactive interference slowing by post-training group

There was weak evidence that MRT was shorter for the Control group than the Meditation group at early training $\left(\mathrm{BF}_{10}\right.$ $=0.56, \mathrm{~W}=48, \hat{\mathrm{R}}=1.0)$ and end of training $\left(\mathrm{BF}_{10}=\right.$ $1.22, \mathrm{~W}=32, \hat{\mathrm{R}}=1.0)$. There was decisive evidence that both groups exhibited improvements in MRT across training $\left(\mathrm{BF}_{10}=4525.65, \mathrm{~W}=210, \hat{\mathrm{R}}=1.8\right)$ but there was weak evidence that groups differed with respect to training improvement $\left(\mathrm{BF}_{10}=0.43, \mathrm{~W}=60, \hat{\mathrm{R}}=1.0\right)$, see Table 2 . Based on moderate evidence, post-training Meditation exhibited less proactive interference in MRT at the introduction of the interference sequence $\left(\mathrm{BF}_{10}=6.61, \mathrm{~W}=84, \hat{\mathrm{R}}=1.0\right)$, see Table 2 . There was moderate evidence that MRT slowing exhibited by the Meditation group was greater than $0\left(\mathrm{BF}_{10}=10.06, \mathrm{~W}=\right.$ $49, \hat{\mathrm{R}}=1.0$ ), while there was decisive evidence that the Control group MRT slowing was greater than $0\left(\mathrm{BF}_{10}=152.10\right.$, $\mathrm{W}=55, \hat{\mathrm{R}}=1.03$ ).

Post-training task manipulation check and subjective ratings There was decisive evidence that more participants who completed the Meditation task self-reported paying attention $\left(\mathrm{N}_{\text {attention }}=9, \mathrm{~N}_{\text {listening }}=1\right)$ than participants who completed the Control audio-listening task $\left(\mathrm{N}_{\text {attention }}=1, \mathrm{~N}_{\text {listening }}=9\right.$, $\mathrm{BF}_{10}=320.65$ ). There was only anecdotal evidence that posttraining groups differed with respect to VAS scores for Distraction $\left(\mathrm{BF}_{10}=1.06, \mathrm{~W}=71.5, \hat{\mathrm{R}}=1.0\right)$ or Effort $\mathrm{BF}_{10}=$ $1.38, \mathrm{~W}=25.5, \hat{\mathrm{R}}=1.0$ ), and weak evidence for group differ- ences in Motivation VAS scores $\mathrm{BF}_{10}=0.41, \mathrm{~W}=46.5, \hat{\mathrm{R}}=$ 1.0) during completion of their respective post-training task.

Wakeful and post-sleep motor memory consolidation That post-training Meditation enhanced wakeful motor memory consolidation to a greater extent than the Control condition was supported by moderate evidence $\left(\mathrm{BF}_{10}=8.34, \mathrm{~W}=87\right.$, $\hat{R}=1.0$ ), see Table 3 . There was moderate evidence that MRT improved for the Meditation group across the wakeful period $\left(\mathrm{BF}_{10}=9.33, \mathrm{~W}=6, \hat{\mathrm{R}}=1.0\right)$, and no evidence that the Control group exhibited wakeful MRT improvement $\left(\mathrm{BF}_{10}=\right.$ $0.12, \mathrm{~W}=46, \hat{\mathrm{R}}=1.0$ ).

Only anecdotal evidence was observed for group differences in time in bed between Meditation $(\mathrm{M}=7.3, \mathrm{SD}=1.0)$ and Control $(\mathrm{M}=7.6, \mathrm{SD}=0.7)$ groups $\left(\mathrm{BF}_{10}=0.49\right.$, error $\%$ $\left.=2.820^{\mathrm{e}-4}\right)$. Similarly, anecdotal evidence was observed for group differences in VAS scores for sleep quality between Meditation $(\mathrm{M}=86.5, \mathrm{SD}=10.6)$ and Control $(\mathrm{M}=75.7$, $\mathrm{SD}=16.5)$ groups $\left(\mathrm{BF}_{10}=1.1\right.$, error $\left.\%=.006\right)$. There was no evidence for group differences in post-sleep consolidation from wakeful test $\left(\mathrm{BF}_{10}=0.38, \mathrm{~W}=48, \hat{\mathrm{R}}=1.0\right)$, despite the Meditation group showing slightly better improvement, see Table 3. There was no evidence of MRT improvement between wakeful test and post-sleep test for the Meditation $\left(\mathrm{BF}_{10}=0.34, \mathrm{~W}=29, \hat{\mathrm{R}}=1.0\right)$ and Control $\mathrm{BF}_{10}=0.28, \mathrm{~W}$ $=31, \hat{\mathrm{R}}=1.0$ ) groups.

Strategy, Pattern, Generation Confidence, Sequence Generation Recall and Recognition Analysis

Four Meditation group participants (40\%) and 3 Control group participants (30\%) reported awareness of a sequence pattern as part of their performance strategy while 6 Meditation group participants (60\%) and 7 Control group participants $(70 \%)$ did not report the presence of a pattern. There was no evidence that groups differed with respect to the distribution of pattern awareness as a performance strategy $\left(\mathrm{BF}_{10}=0.53\right)$. There was also no evidence of group differences in the distribution of participants who identified that order of stimuli as following a sequence (Meditation $=2$, Control $=0$ ) or a random order $($ Meditation $=8$, Control $=$ $10, \mathrm{BF}_{10}=0.73$ ).

VAS scores for confidence in generating the sequence were not different between Meditation $(\mathrm{M}=37.9, \mathrm{SD}=20.5)$ and Control $(\mathrm{M}=30.2, \mathrm{SD}=20.0)$ groups $\left(\mathrm{BF}_{10}=0.48, \mathrm{~W}=36\right.$, $\hat{\mathrm{R}}=1.0$ ). Groups did not differ with respect to the accuracy percentage of trained sequence generation $\left(\mathrm{BF}_{10}=0.42, \mathrm{~W}=\right.$ 52.5, $\hat{\mathrm{R}}=1.0$ ), see Table 4 . There was no evidence that the 
Table 2. Post-training Control and Meditation group comparisons and non-parametric Bayesian inference results for early training and end training mean RT, and training improvement and interference slowing.

\begin{tabular}{|c|c|c|c|c|c|c|c|c|}
\hline \multirow[b]{3}{*}{ Mean (SD) } & \multicolumn{2}{|c|}{ Early Training Mean RT } & \multicolumn{2}{|c|}{ End Training Mean RT } & \multicolumn{2}{|c|}{ Training Improvement } & \multicolumn{2}{|c|}{ Interference Slowing } \\
\hline & Control & Meditation & Control & Meditation & \begin{tabular}{|l|} 
Control \\
\end{tabular} & Meditation & \begin{tabular}{|l|} 
Control \\
\end{tabular} & Meditation \\
\hline & $387.4(67.0)$ & $433.6(138.1)$ & $285.4(67.6)$ & $333.8(54.8)$ & $101.9(45.2)$ & $99.8(96.9)$ & $75.0(52.6)$ & $25.5(30.7)$ \\
\hline $95 \% \mathrm{Cl}$ & {$[339.4,435.3]$} & {$[334.9,532.4]$} & {$[237.1,333.8]$} & {$[294.7,373.0]$} & {$[69.6,134.2]$} & {$[30.5,169.1]$} & {$[37.3,112.6]$} & {$[3.5,47.4]$} \\
\hline Alternate hypothes & \multicolumn{2}{|c|}{ Control $<$ Meditation } & \multicolumn{2}{|c|}{ Control $<$ Meditation } & \multicolumn{2}{|c|}{ Control $=$ Meditation } & \multicolumn{2}{|c|}{ Control $>$ Meditation } \\
\hline $\mathrm{BF}_{10}$ & \multicolumn{2}{|c|}{0.56} & \multicolumn{2}{|c|}{1.22} & \multicolumn{2}{|c|}{0.43} & \multicolumn{2}{|c|}{6.61} \\
\hline $\begin{array}{l}\text { Effect Size Median } \\
\delta(95 \% \mathrm{Cl})\end{array}$ & \multicolumn{2}{|c|}{$-0.30(-0.98,-0.015)$} & \multicolumn{2}{|c|}{$-0.47(-1.3,-0.032)$} & \multicolumn{2}{|c|}{$0.11(-0.64,0.90)$} & \multicolumn{2}{|c|}{$0.89(0.13,1.86)$} \\
\hline Cohen's d & \multicolumn{2}{|c|}{-0.43} & \multicolumn{2}{|c|}{-0.79} & \multicolumn{2}{|c|}{0.028} & \multicolumn{2}{|c|}{1.1} \\
\hline
\end{tabular}

Table 3. Post-training Control and Meditation group comparisons and nonparametric Bayesian inference results for changes in mean RT associated with wakeful and sleep-dependent forms of motor memory consolidation.

\begin{tabular}{|c|c|c|c|c|}
\hline \multirow[b]{2}{*}{ Mean (SD) } & \multicolumn{2}{|c|}{$\begin{array}{c}\text { Training to Wakeful Test } \\
\text { Consolidation }\end{array}$} & \multicolumn{2}{|c|}{$\begin{array}{c}\text { Wakeful Test to Post-Sleep } \\
\text { Test Consolidation }\end{array}$} \\
\hline & $\begin{array}{c}\text { Control } \\
28.6(47.7)\end{array}$ & $\begin{array}{l}\text { Meditation } \\
-22.1(23.0)\end{array}$ & \begin{tabular}{|c|} 
Control \\
$2.2(22.8)$
\end{tabular} & $\begin{array}{c}\text { Meditation } \\
1.0(29.0)\end{array}$ \\
\hline $95 \% \mathrm{Cl}$ & {$[-5.6,62.7]$} & {$[-38.6,-5.7]$} & {$[-14.1,18.6]$} & {$[-19.7,21.8]$} \\
\hline Alternate hypothe & \multicolumn{2}{|c|}{ Control > Meditation } & \multicolumn{2}{|c|}{ Control > Meditation } \\
\hline $\mathrm{BF}_{10}$ & \multicolumn{2}{|c|}{8.34} & \multicolumn{2}{|c|}{0.38} \\
\hline $\begin{array}{l}\text { Effect Size } \\
\text { Median } \delta(95 \% \mathrm{Cl})\end{array}$ & \multicolumn{2}{|c|}{$0.96(0.15,1.96)$} & \multicolumn{2}{|c|}{$0.22(0.0080,0.82)$} \\
\hline Cohen's d & \multicolumn{2}{|c|}{1.35} & \multicolumn{2}{|c|}{0.046} \\
\hline
\end{tabular}

recall accuracy rate was above chance level for Meditation $\left(\mathrm{BF}_{10}=0.10, \mathrm{~W}=22, \hat{\mathrm{R}}=1.1\right)$ and Control $\mathrm{BF}_{10}=0.13, \mathrm{~W}$ $=11.5, \hat{\mathrm{R}}=1.1)$ groups.

There was no evidence that groups differed with respect to recognition performance on trained sequence chunk hits $\left(\mathrm{BF}_{10}=0.61, \mathrm{~W}=37, \hat{\mathrm{R}}=1.0\right)$, interference sequence chunk hits $\mathrm{BF}_{10}=0.57, \mathrm{~W}=33.5, \hat{\mathrm{R}}=1.0$ ), or novel sequence chunk correct rejections $\mathrm{BF}_{10}=0.48, \mathrm{~W}=58, \hat{\mathrm{R}}=1.0$ ). Moreover, there was no evidence that overall recognition performance was above chance level for Meditation $\left(\mathrm{BF}_{10}=0.08, \mathrm{~W}=44\right.$, $\hat{\mathrm{R}}=2.4$ ) and Control $\mathrm{BF}_{10}=0.13, \mathrm{~W}=55, \hat{\mathrm{R}}=1.1$ ) groups, see Table 4.

\section{Discussion}

We investigated the effect of single-session mindfulness meditation on wakeful and sleep-dependent forms of implicit motor memory consolidation with naïve meditators. Participants first completed training on the SRTT and then completed 10 minutes of either meditation or audiobook listening, before being exposed to SRTT interference. A wakeful period of 5 hours followed before completing testing during the posttraining wakeful period in addition to completing a post-sleep test the following day. A single session of meditation appeared to promote post-training wakeful motor memory consolidation as illustrated by strong evidence that meditation resulted in MRT improvement between the end of training and the start of wakeful test in comparison to control. Sleepdependent motor consolidation did not appear to benefit from post-training meditation as there were no significant group differences in MRT performance between the end of wakeful test, and the start of post-sleep test.

The present demonstration of improved wakeful consolidation from post-training meditation is consistent with Immink
(2016). However, the present results are more aligned with wakeful offline improvements whereas Immink (2016) reported only wakeful stabilization. The use of an implicit motor task here might explain this discrepancy, given that implicit sequence learning exhibits wakeful offline learning (Robertson, Pascual-Leone, \& Miall, 2004), whereas explicit sequence learning relies on sleep for offline improvements (Walker et al., 2002). However, other demonstrations of wakeful motor memory consolidation associated with exercise have shown that explicit sequences can too provide for wakeful offline improvements (Rhee et al., 2016). This discrepancy might provide a case for the interaction of explicit and implicit learning during motor task acquisition, instead of being distinct forms of learning (Immink, 2016). Willingham and Goedert-Eschmann (1999) suspect implicit knowledge is acquired in parallel with explicit knowledge given their observation of equivalent motor sequence learning outcomes. However, although shared networks were identified, Sami et al. (2014) report largely distinct neural mechanisms under implicit and explicit conditions across a 6 hour resting-state consolidation period. Contradictory findings such as these indicate that further research is required to distinguish the effects of task awareness on motor skill learning during both wakeful and post-sleep periods (Song, 2009). Also distinct from Immink (2016), we show that meditation experience is not necessarily required to support motor memory consolidation, as evidenced by meditation naïve individuals exhibiting wakeful consolidation. Previous work has shown that the executive attention benefit afforded by extensive meditation training (Jha et al., 2007) extends to even single sessions of meditation practice through improving the allocation of attentional resources in novices (Norris et al., 2018). This is reinforced with previous demonstrations that meditation naïve individuals practicing a single session of FAM are able to exhibit a level of cognitive control that led to performance improvements on the SRTT (Chan et al., 2017).

The absence of group differences in post-sleep consolidation indicates that post-training meditation effects on motor consolidation are limited to wakeful consolidation processes. Previous work has shown that focus of attention during the execution of a motor task supports superior performance (Wulf \& Prinz, 2001). Duke et al. (2011) had learners perform a simple piano keyboard passage and were instructed to focus their attention to either their fingers, the piano keys, the piano hammers, or the ensuing sound of the 
Table 4. Post-training Control and Meditation group comparisons and non-parametric Bayesian inference results for recall accuracy of the generated trained sequence and recognition accuracy based on correct identification, or hits, of sequence chunks from trained and interference sequences and correct rejection of novel sequence chunks.

\begin{tabular}{|c|c|c|c|c|c|c|c|c|}
\hline \multirow[b]{2}{*}{ Mean (SD) } & \multicolumn{2}{|c|}{ Sequence Generation Recall \% } & \multicolumn{2}{|c|}{$\begin{array}{l}\text { Trained Sequence } \\
\text { Recognition, Hit \% }\end{array}$} & \multicolumn{2}{|c|}{$\begin{array}{l}\text { Interference Sequence } \\
\text { Recognition, Hit \% }\end{array}$} & \multicolumn{2}{|c|}{$\begin{array}{c}\text { Novel Sequence Recognition, } \\
\text { Correct Rejection } \%\end{array}$} \\
\hline & $\begin{array}{c}\text { Control } \\
29.2(10.6)\end{array}$ & $\begin{array}{l}\text { Meditation } \\
29.2(11.3)\end{array}$ & $\begin{array}{c}\text { Control } \\
62.5(12.6)\end{array}$ & $\begin{array}{l}\text { Meditation } \\
69.2(14.7)\end{array}$ & \begin{tabular}{c|} 
Control \\
$55.8(15.2)$
\end{tabular} & $\begin{array}{l}\text { Meditation } \\
61.7(11.9)\end{array}$ & \begin{tabular}{c|} 
Control \\
$59.2(13.2)$
\end{tabular} & $\begin{array}{l}\text { Meditation } \\
54.2(15.3)\end{array}$ \\
\hline $95 \% \mathrm{Cl}$ & {$[339.4,435.3]$} & {$[334.9,532.4]$} & {$[237.1,333.8]$} & {$[294.7,373.0]$} & {$[69.6,134.2]$} & {$[30.5,169.1]$} & {$[37.3,112.6]$} & {$[3.5,47.4]$} \\
\hline Alternate hypothes & \multicolumn{2}{|c|}{ Control $\neq$ Meditation } & \multicolumn{2}{|c|}{ Control $\neq$ Meditation } & \multicolumn{2}{|c|}{ Control $=$ Meditation } & \multicolumn{2}{|c|}{ Control $\neq$ Meditation } \\
\hline $\mathrm{BF}_{10}$ & \multicolumn{2}{|c|}{0.42} & \multicolumn{2}{|c|}{0.69} & \multicolumn{2}{|c|}{0.57} & \multicolumn{2}{|c|}{0.48} \\
\hline $\begin{array}{l}\text { Effect Size Median } \\
\delta(95 \% \mathrm{Cl})\end{array}$ & \multicolumn{2}{|c|}{$0.015(-0.73,0.80)$} & \multicolumn{2}{|c|}{$-0.33(-1.21,0.39)$} & \multicolumn{2}{|c|}{$-0.31(-1.17,0.41)$} & \multicolumn{2}{|c|}{$0.20(-0.55,1.00)$} \\
\hline Cohen's d & \multicolumn{2}{|c|}{-0.00018} & \multicolumn{2}{|c|}{-0.49} & \multicolumn{2}{|c|}{-0.43} & \multicolumn{2}{|c|}{-0.35} \\
\hline
\end{tabular}

key presses. Learners who focused their attention on the effects of their movements (i.e. the sound produced) compared to the movements themselves exhibited more accurate motor control (Duke et al., 2011). Similarly, meditation as a posttraining cognitive task might support wakeful motor memory consolidation through the manner in which meditation techniques establish heightened attention control (Norris et al., 2018), resulting in enhanced memory formation (Duke et al., 2011). Previous research has shown that SRTT performance is influenced by a preceding session of FAM via an increased level of top-down cognitive control afforded by FAM (Chan et al., 2017). Given consolidation processes occur following training, the attention control benefit through meditation might play a role in the strengthening of motor memories in the form of wakeful offline learning as evidenced by the present demonstration.

Post-training meditation also reduced negative transfer between training and interference sequences as evidenced by a reduced MRT change from late training to early interference. A secondary task variation is used following training to disrupt attention (Song, 2009), and thus a post-training state comprised of attention captured in meditation was able to lessen this disruption. Similarly, previous research found exposure to exercise following training reduced the impact of the interfering sequence which was manifested in the form of offline motor sequence improvements (Rhee et al., 2016). The demonstrations of resistance to interference leading to offline gain exhibited by both exercise and meditation interventions reinforce results by Austin and Loprinzi (2019), where coupling post-training exercise and meditation led to superior long term memory on a declarative task. A synergistic effect of exercise and meditation might also be observed for the consolidation of motor skills. The shared activity in the DLPFC during both exercise (Yanagisawa et al., 2010) and meditation (Cahn \& Polich, 2006) is one mechanism that might help to lessen the effect of interference given its association with focused attention (Hasenkamp et al., 2012). In the context of meditation, DLPFC activity is thought to repeatedly redirect or sustain attention (Lippelt et al., 2014), and similarly, DLPFC activity with exercise has been linked to heightened concentration and mental focus following exercise (Moriarty et al., 2019). Research including motor skill training followed by meditation and exercise interventions is required to provide further clarification if this effect does indeed translate to motor skill consolidation processes.
Despite post-training meditation failing to establish a distinct benefit for sleep-dependent motor consolidation as compared to control, as expected, the offline improvements in both groups exhibited from training to post-sleep test supports a wealth of research in favor of sleep-dependent gains in performance (Diekelmann \& Born, 2010). As mentioned, research by Solomonova et al. (2020) investigated whether meditation practice changes the neurobiological qualities of sleep-dependent memory consolidation by comparing the performance of experienced Vipassana meditators with meditation naïve individuals on a procedural balance task. Though both groups experienced improved performance as anticipated via sleep, the way in which these improvements arose were distinct. The experienced meditators improvements were correlated with occipital spindle density in non-rapid eye movement (NREM) sleep, however the controls task improvements were correlated with increased time in rapid eye movement (REM) sleep (Solomonova et al., 2020). The authors note that previous training on attending to bodily states and stimuli might generate a different neurobiological way of learning which might help explain previous findings showing an enhanced level of attention is associated with improved motor performance (Hazeltine et al., 1997). Given these findings pertain to the way in which experienced meditators exhibit performance improvements, further research is needed to determine if this effect translates to a single session of meditation following training as in context of the present research.

Limitations and Future Research

The present demonstration of wakeful motor memory consolidation is based on a small sample of 10 meditation naïve individuals (Lohse et al., 2016) which may have resulted in insufficient statistical power (Rhee et al., 2016). However, it is thought that using Bayesian estimation is generally effective in preventing the overestimation in favor of an effect (Wetzels et al., 2011), particularly in the instance of small samples (McNeish, 2016).

The extent to which novice meditators were able to stay on task during meditation remains to be determined. Past research indicates that novices can improve attention in a brief 10-minute mindfulness meditation session (Norris et al., 2018). However further investigation using EEG to clarify the neural markers of meditation in novices is required given the large variance of EEG signals achieved by novice meditators (Stapleton et al., 2020). Though beyond the scope of 
the present experiment, accurately describing the neural correlates of meditation in novices is an important next step to work toward determining whether meditation-induced motor memory consolidation is a by-product of achieving a state of post-training attention control.

The present findings were established in the context of a fine motor skill task in the SRTT (Nissen \& Bullemer, 1987). Lugassy et al. (2018) has reported that the mechanisms in complex motor tasks operate distinctly to that of simple motor tasks by way of delayed offline consolidation in more complex skill learning. Given these motor skill differences, further work is needed to determine if meditation-induced consolidation effects are able to translate to the learning of more complex motor skill task variations involving gross body representation to expand the applications in which this intervention is applicable (Lugassy et al., 2018). By extension, whether the motor task is implicitly or explicitly learned also needs to be determined in the context of post-training interventions involving meditation. As noted earlier, previous findings by Immink (2016) demonstrated memory stabilization form of consolidation using an explicit sequence learning task, and compared to the present findings of offline improvements using an implicit SRTT, highlights that task awareness might be important to consider for such interventions involving meditation.

The present results demonstrate that a single post-training meditation session with novice mediators can support motor memory consolidation in the context of offline improvements. Importantly, these offer important contributions toward understanding wakeful motor memory consolidation processes. This is highlighted by: 1) heightened posttraining attention control is important for reducing negative transfer to new task variations, 2) heightened post-training attention control from meditation enhances wakeful but not sleep-dependent motor consolidation, and 3) wakeful consolidation gains from meditation induced attention control do not require extensive mindfulness training.

\section{ACKNOWLEDGEMENTS}

No funding was received for conducting this study.

Informed consent was obtained from all individual participants included in the study. All procedures performed involving human participants were in accordance with the principles of the Declaration of Helsinki. The study was approved by the University of South Australia Human Research Ethics Committee (protocol number 202937). The authors have no relevant financial or non-financial interests to disclose.

\section{References}

Atkinson, W. (1911). Your Mind and How to Use it: A Manual of Practical Psychology (Vol. Volume 1 of Library of Alexandria). Library of Alexandria.

Austin, M., \& Loprinzi, P. D. (2019). Acute exercise and mindfulness meditation on learning and memory: randomized controlled intervention. Health Promotion Perspectives, 9(4), 314-318. https://doi.org/10.15171/hpp.2019.43

Baerentsen, K. B., Hartvig, N. V., Stødkilde-Jørgensen, H., \& Mammen, J. (2001). Onset of meditation explored with fMRI. NeuroImage, 13(6), 297. https://doi.org/10.1016/s1053-8119(01)91640-4

Baijal, S., \& Srinivasan, N. (2010). Theta activity and meditative states: spectral changes during concentrative meditation. Cognitive Processing, 11(1), 31-38. https://doi.org/10.1007/s10339-009-0272-0

Black, D. S., Sussman, S., Johnson, C. A., \& Milam, J. (2012). Psychometric Assessment of the Mindful Attention Awareness Scale (MAAS) Among Chinese Adolescents. Assessment, 19(1), 42-52. https://doi.org/10.1177/1073191111415365
Brokaw, K., Tishler, W., Manceor, S., Hamilton, K., Gaulden, A., Parr, E., \& Wamsley, E. J. (2016). Resting state EEG correlates of memory consolidation. Neurobiology of Learning and Memory, 130, 17-25. https://doi.org/10.1016/j.nlm.2016.01.008

Brown, K. W., \& Ryan, R. M. (2003). The benefits of being present: Mindfulness and its role in psychological well-being. Journal of Personality and Social Psychology, 84(4), 822-848. https://doi.org/10.1037/00223514.84.4.822

Brown, K. W., West, A. M., Loverich, T. M., \& Biegel, G. M. (2011). Assessing adolescent mindfulness: Validation of an Adapted Mindful Attention Awareness Scale in adolescent normative and psychiatric populations. 23(4), 1023-1033. https://doi.org/10.1037/a0021338

Brown, R., \& Robertson, E. (2007). Inducing motor skill improvements with a declarative task. Nature Neuroscience, 10(2), 148-149. https://doi.org/10.1038/nn1836

Buysse, D. J., Reynolds, C. F., Monk, T. H., Berman, S. R., \& Kupfer, D. J. (1989). The Pittsburgh sleep quality index: A new instrument for psychiatric practice and research. Psychiatry Research, 28(2), 193-213. https://doi.org/10.1016/0165-1781(89)90047-4

Cahn, B. R., \& Polich, J. (2006). Meditation states and traits: EEG, ERP, and neuroimaging studies. Psychological bulletin, 132(2), 180-211. https://doi.org/10.1037/0033-2909.132.2.180 Carlson, L. E., \& Brown, K. W. (2005). Validation of the Mindful Attention Awareness Scale in a cancer population. Journal of Psychosomatic Research, 58(1), 29-33. https://doi.org/10.1016/j.jpsychores.2004.04.366

Chan, R. W., Alday, P. M., Zou-Williams, L., Lushington, K., Schlesewsky, M., Bornkessel-Schlesewsky, I., \& Immink, M. A. (2020). Focused-attention meditation increases cognitive control during motor sequence performance: Evidence from the N2 cortical evoked potential. Behavioural Brain Research, 384, 112536. https://doi.org/10.1016/j.bbr.2020.112536

Chan, R. W., Immink, M. A., \& Lushington, K. (2017). The influence of focused-attention meditation states on the cognitive control of sequence learning. Consciousness and Cognition, 55, 11-25. https://doi.org/10.1016/j.concog.2017.07.004

Chan, R. W., Lushington, K., \& Immink, M. A. (2018). States of focused attention and sequential action: A comparison of single session meditation and computerised attention task influences on top-down control during sequence learning. Acta Psychologica, 191, 87-100. https://doi.org/10.1016/j.actpsy.2018.09.003

Chang, Y. K., Labban, J. D., Gapin, J. I., \& Etnier, J. L. (2012). The effects of acute exercise on cognitive performance: A meta-analysis. Brain Research, 1453, 87-101. https://doi.org/10.1016/j.brainres.2012.02.068

Colcombe, S., \& Kramer, A. F. (2003). Fitness Effects on the Cognitive Function of Older Adults. Psychological Science, 14(2), 125-130. https://doi.org/10.1111/1467-9280.t01-1-01430

Colzato, L., Szapora Ozturk, A., Pannekoek, J., \& Hommel, B. (2013). The impact of physical exercise on convergent and divergent thinking [Original Research]. Frontiers in Human Neuroscience, 7(824). https://doi.org/10.3389/fnhum.2013.00824

Colzato, L. S., Szapora, A., Lippelt, D., \& Hommel, B. (2017). Prior meditation practice modulates performance and strategy use in convergent- and divergent-thinking problems. Mindfulness, 8(1), 10-16. https://doi.org/10.1007/s12671-014-0352-9

Dahms, C., Brodoehl, S., Witte, O. W., \& Klingner, C. M. (2020). The importance of different learning stages for motor sequence learning after stroke. Human Brain Mapping, 41(1), 270-286. https://doi.org/10.1002/hbm.24793

Davidson, R., \& Kaszniak, A. (2015). Conceptual and Methodological Issues in Research on Mindfulness and Meditation. American Psychologist, 70(7), 581-592. https://doi.org/10.1037/a0039512

Dayan, E., \& Cohen, L. (2011). Neuroplasticity Subserving Motor Skill Learning. Neuron, 72(3), 443-454. https://doi.org/10.1016/j.neuron.2011.10.008

De Bruin, E. I., Van Der Zwan, J. E., \& Bögels, S. M. (2016). A RCT Comparing Daily Mindfulness Meditations, Biofeedback Exercises, and Daily Physical Exercise on Attention Control, Executive Functioning, Mindful Awareness, Self-Compassion, and Worrying in Stressed Young Adults. Mindfulness, 7(5), 1182-1192. https://doi.org/10.1007/s12671016-0561-5

Debas, K., Carrier, J., Orban, P., Barakat, M., Lungu, O., Vandewalle, G., . . . Doyon, J. (2010). Brain plasticity related to the consolidation of motor sequence learning and motor adaptation. Proceedings of the National Academy of Sciences, 107(41), 17839-17844. https://doi.org/10.1073/pnas.1013176107

Diekelmann, S., \& Born, J. (2007). One memory, two ways to consolidate? Nature Neuroscience, 10(9), 1085-1086. https://doi.org/10.1038/nn0907-1085

Diekelmann, S., \& Born, J. (2010). The memory function of sleep. Nature Reviews Neuroscience, 11(2), 114-126. https://doi.org/10.1038/nrn2762

Duke, R. A., Cash, C. D., \& Allen, S. E. (2011). Focus of Attention Affects Performance of Motor Skills in Music. Journal of Research in Music Education, 59(1), 44-55. https://doi.org/10.1177/0022429410396093

Dumel, G., Bourassa, M. E., Desjardins, M., Voarino, N., CharleboisPlante, C., Doyon, J., \& De Beaumont, L. (2016). Multisession Anodal tDCS Protocol Improves Motor System Function in 
an Aging Population. Neural plasticity, 2016, 5961362-5961362. https://doi.org/10.1155/2016/5961362

Etnier, J. L., Salazar, W., Landers, D. M., Petruzzello, S. J., Han, M., \& Nowell, P. (1997). The Influence of Physical Fitness and Exercise upon Cognitive Functioning: A Meta-Analysis. Journal of Sport and Exercise Psychology, 19(3), 249-277. https://doi.org/10.1123/jsep.19.3.249

Fischer, S., Hallschmid, M., Elsner, A. L., \& Born, J. (2002). Sleep forms memory for finger skills. Proceedings of the National Academy of Sciences, 99(18), 11987-11991. https://doi.org/10.1073/pnas.182178199

Hasenkamp, W., Wilson-Mendenhall, C. D., Duncan, E., \& Barsalou, L. W. (2012). Mind wandering and attention during focused meditation: A fine-grained temporal analysis of fluctuating cognitive states. NeuroImage, 59(1), 750-760. https://doi.org/https://doi.org/10.1016/j.neuroimage.2011.07.008

Hazeltine, E., Grafton, S. T., \& Ivry, R. (1997). Attention and stimulus characteristics determine the locus of motor- sequence encoding. A PET study. Brain, 120(1), 123-140. https://doi.org/10.1093/brain/120.1.123

Hertel, P. T., \& Rude, S. S. (1991). Depressive deficits in memory: Focusing attention improves subsequent recall. Journal of Experimental Psychology: General, 120(3), 301-309. https://doi.org/10.1037/00963445.120.3.301

Heyes, C. M., \& Foster, C. L. (2002). Motor learning by observation: Evidence from a serial reaction time task. The Quarterly Journal of Experimental Psychology Section A, 55(2), 593-607. https://doi.org/10.1080/02724980143000389

Immink, M. A. (2016). Post-training Meditation Promotes Motor Memory Consolidation. Frontiers in Psychology, 7(1698), 1-10. https://doi.org/10.3389/fpsyg.2016.01698

Immink, M. A., Colzato, L. S., Stolte, M., \& Hommel, B. (2017). Sequence Learning Enhancement Following Single-Session Meditation Is Dependent on Metacontrol Mode and Experienced Effort. Journal of Cognitive Enhancement, 1(2), 127-140. https://doi.org/10.1007/s41465-017-00192

Jha, A. P., Krompinger, J., \& Baime, M. J. (2007). Mindfulness training modifies subsystems of attention. Cognitive, Affective, \& Behavioral Neuroscience, 7(2), 109-119. https://doi.org/10.3758/cabn.7.2.109

King, B. R., Hoedlmoser, K., Hirschauer, F., Dolfen, N., \& Albouy, G. (2017). Sleeping on the motor engram: The multifaceted nature of sleeprelated motor memory consolidation. Neuroscience \& Biobehavioral Reviews, 80, 1-22. https://doi.org/10.1016/j.neubiorev.2017.04.026

Korman, M., Doyon, J., Doljansky, J., Carrier, J., Dagan, Y., \& Karni, A. (2007). Daytime sleep condenses the time course of motor memory consolidation. Nature Neuroscience, 10(9), 1206-1213. https://doi.org/10.1038/nn1959

Lambourne, K., \& Tomporowski, P. (2010). The effect of exercise-induced arousal on cognitive task performance: A meta-regression analysis. Brain Research, 1341, 12-24. https://doi.org/10.1016/j.brainres.2010.03.091

Larson, M. J., Steffen, P. R., \& Primosch, M. (2013). The impact of a brief mindfulness meditation intervention on cognitive control and errorrelated performance monitoring. Frontiers in Human Neuroscience, 7. https://doi.org/10.3389/fnhum.2013.00308

Lazar, S., Bush, G., Gollub, R., Fricchione, G., Khalsa, G., \& Benson, H. (2000). Functional brain mapping of the relaxation response and meditation. NeuroReport, 11, 1581-1585. https://doi.org/https://doi.org/10.1097/00001756-200005150-00042

Lee, Kulubya, E., Goldin, P., Goodarzi, A., \& Girgis, F. (2018). Review of the Neural Oscillations Underlying Meditation. Frontiers in Neuroscience, 12, 178-178. https://doi.org/10.3389/fnins.2018.00178

Levinson, D., Stoll, E., Kindy, S., Merry, H., \& Davidson, R. (2014). A mind you can count on: validating breath counting as a behavioural measure of mindfulness. Frontiers in Psychology, 5(1202), 1-10. https://doi.org/10.3389/fpsyg.2014.01202/full

LimeSurvey. (2020). LimeSurvey: An Open Source survey tool. LimeSurvey GmBH. http://www.limesurvey.org

Lippelt, D. P., Hommel, B., \& Colzato, L. S. (2014). Focused attention, open monitoring and loving kindness meditation: effects on attention, conflict monitoring, and creativity â€“" A review. Frontiers in Psychology, 5. https://doi.org/10.3389/fpsyg.2014.01083

Lohse, K., Buchanan, T. L., \& Miller, M. (2016). Underpowered and Overworked: Problems With Data Analysis in Motor Learning Studies. Journal of Motor Learning and Development, 4, 37-58. https://doi.org/https://doi.org/10.1123/JMLD.2015-0010

Lugassy, D., Herszage, J., Pilo, R., Brosh, T., \& Censor, N. (2018). Consolidation of complex motor skill learning: evidence for a delayed offline process. Sleep, 41(9). https://doi.org/10.1093/sleep/zsy123

Lumma, A.-L., Kok, B. E., \& Singer, T. (2015). Is meditation always relaxing? Investigating heart rate, heart rate variability, experienced effort and likeability during training of three types of meditation. International Journal of Psychophysiology, 97(1), 38-45. https://doi.org/10.1016/j.ijpsycho.2015.04.017

Maclean, K. A., Ferrer, E., Aichele, S. R., Bridwell, D. A., Zanesco, A. P., Jacobs, T. L., . . . Saron, C. D. (2010). Intensive Meditation Training Improves Perceptual Discrimination and Sustained Attention. Psychological Science, 21(6), 829-839. https://doi.org/10.1177/0956797610371339
Makowski, D., Ben-Shachar, M. S., Chen, S. H. A., \& Lüdecke, D. (2019). Indices of Effect Existence and Significance in the Bayesian Framework. Frontiers in Psychology, 10. https://doi.org/10.3389/fpsyg.2019.02767

Malinowski, P. (2013). Neural mechanisms of attentional control in mindfulness meditation. Frontiers in Neuroscience, 7. https://doi.org/10.3389/fnins.2013.00008

Marcora, S. M., Staiano, W., \& Manning, V. (2009). Mental fatigue impairs physical performance in humans. Journal of Applied Physiology, 106(3), 857-864. https://doi.org/10.1152/japplphysiol.91324.2008

McAuley, E., Mullen, S. P., Szabo, A. N., White, S. M., Wójcicki, T. R., Mailey, E. L., . . . Kramer, A. F. (2011). Self-Regulatory Processes and Exercise Adherence in Older Adults. American Journal of Preventive Medicine, 41(3), 284-290. https://doi.org/10.1016/j.amepre.2011.04.014

McNeish, D. (2016). On Using Bayesian Methods to Address Small Sample Problems. Structural Equation Modeling A Multidisciplinary Journal, 23. https://doi.org/10.1080/10705511.2016.1186549

Mednick, S., Nakayama, K., \& Stickgold, R. (2003). Sleep-dependent learning: a nap is as good as a night. Nature Neuroscience, 6(7), 697-698. https://doi.org/10.1038/nn1078

Moore, A., Gruber, T., Derose, J., \& Malinowski, P. (2012). Regular, brief mindfulness meditation practice improves electrophysiological markers of attentional control [Original Research]. Frontiers in Human Neuroscience, 6(18). https://doi.org/10.3389/fnhum.2012.00018

Moriarty, T., Bourbeau, K., Bellovary, B., \& Zuhl, M. N. (2019). Exercise Intensity Influences Prefrontal Cortex Oxygenation during Cognitive Testing. Behavioral Sciences, 9(8), 83 https://doi.org/10.3390/bs9080083

Mrazek, M., Franklin, M. S., Phillips, D. T., Baird, B., \& Schooler, J. W. (2013). Mindfulness Training Improves Working Memory Capacity and GRE Performance While Reducing Mind Wandering. Psychological Science, 24(5), 776-781. https://doi.org/10.1177/0956797612459659

Mrazek, M., Smallwood, J., \& Schooler, J. (2012). Mindfulness and MindWandering: Finding Convergence Through Opposing Constructs. Emotion, 12(3), 442-448. https://doi.org/10.1037/a0026678

Newberg, A., Alavi, A., Baime, M., Pourdehnad, M., Santanna, J., \& D'Aquili, E. (2001). The measurement of regional cerebral blood flow during the complex cognitive task of meditation: a preliminary SPECT study. Psychiatry Research: Neuroimaging, 106(2), 113-122. https://doi.org/10.1016/s0925-4927(01)00074-9

Nishida, M., \& Walker, M. P. (2007). Daytime Naps, Motor Memory Consolidation and Regionally Specific Sleep Spindles. PLoS ONE 2(4), e341. https://doi.org/10.1371/journal.pone.0000341 Nissen, M. J., \& Bullemer, P. (1987). Attentional requirements of learning: Evidence from performance measures. Cognitive Psychology, 19(1), 1-32. https://doi.org/10.1016/0010-0285(87)90002-8

Norris, C. J., Creem, D., Hendler, R., \& Kober, H. (2018). Brief Mindfulness Meditation Improves Attention in Novices: Evidence From ERPs and Moderation by Neuroticism. Frontiers in Human Neuroscience, 12 https://doi.org/10.3389/fnhum.2018.00315

Osman, A., Lamis, D. A., Bagge, C. L., Freedenthal, S., \& Barnes, S. M. (2016). The Mindful Attention Awareness Scale: Further Examination of Dimensionality, Reliability, and Concurrent Validity Estimates. Journal of Personality Assessment, 98(2), 189-199. https://doi.org/10.1080/00223891.2015.1095761

Reed, J., \& Johnson, P. (1994). Assessing implicit learning with indirect tests: Determining what is learned about sequence structure. Journal of experimental Psychology: Learning, Memory and Cognition, 20(3), 585-594. https://doi.org/10.1037/0278-7393.20.3.585

Rhee, J., Chen, J., Riechman, S. M., Handa, A., Bhatia, S., \& Wright, D. L. (2016). An acute bout of aerobic exercise can protect immediate offline motor sequence gains. Psychological Research, 80(4), 518-531. https://doi.org/10.1007/s00426-015-0682-9

Robertson, E. (2007). The Serial Reaction Time Task: Implicit Motor Skill Learning? Journal of Neuroscience, 27(38), 10073-10075. https://doi.org/10.1523/jneurosci.2747-07.2007

Robertson, E., Pascual-Leone, A., \& Miall, R. C. (2004). Current concepts in procedural consolidation. Nature Reviews Neuroscience, 5(7), 576582. https://doi.org/10.1038/nrn1426

Robertson, E., Pascual-Leone, A., \& Press, D. Z. (2004). Awareness Modifies the Skill-Learning Benefits of Sleep. Current Biology, 14(3), 208212. https://doi.org/10.1016/j.cub.2004.01.027

Roig, M., Skriver, K., Lundbye-Jensen, J., Kiens, B., \& Nielsen, J. B. (2012) A Single Bout of Exercise Improves Motor Memory. PLoS ONE, 7(9), e44594. https://doi.org/10.1371/journal.pone.0044594

Sami, S., Robertson, E. M., \& Miall, R. C. (2014). The Time Course of Task-Specific Memory Consolidation Effects in Resting State Networks. Journal of Neuroscience, 34(11), 3982-3992. https://doi.org/10.1523/jneurosci.4341-13.2014

Schneider, W., Eschman, A., and Zuccolotto, A. (2016). Psychology Software Tools, Inc. [E-Prime 3.0]. Psychology Software Tools Inc.

Schneider, W., Eschman, A., and Zuccolotto, A. (2020). Psychology Software Tools, Inc. [E-Prime Go]. Psychology Software Tools Inc.

Schücker, L., Anheier, W., Hagemann, N., Strauss, B., \& Völker, K. (2013). On the optimal focus of attention for efficient running at high inten- 
sity. Sport, Exercise, and Performance Psychology, 2(3), 207-219. https://doi.org/10.1037/a0031959

Shadmehr, R., \& Brashers-Krug, T. (1997). Functional Stages in the Formation of Human Long-Term Motor Memory. The Journal of Neuroscience, 17(1), 409-419. https://doi.org/10.1523/jneurosci.17-01-00409.1997

Shiffman, S., Stone, A. A., \& Hufford, M. R. (2008). Ecological Momentary Assessment. Annual Review of Clinical Psychology, 4(1), 1-32. https://doi.org/10.1146/annurev.clinpsy.3.022806.091415

Solomonova, E., Dubé, S., Blanchette-Carrière, C., Sandra, D. A., SamsonRicher, A., Carr, M., . . . Nielsen, T. (2020). Different Patterns of SleepDependent Procedural Memory Consolidation in Vipassana Meditation Practitioners and Non-meditating Controls. Frontiers in Psychology, 10. https://doi.org/10.3389/fpsyg.2019.03014

Song, S. (2009). Consciousness and the consolidation of motor learning. Behavioural Brain Research, 196(2), 180-186. https://doi.org/10.1016/j.bbr.2008.09.034

Stapleton, P., Dispenza, J., McGill, S., Sabot, D., Peach, M., \& Raynor, D. (2020). Large effects of brief meditation intervention on EEG spectra in meditation novices. IBRO Reports, 9, 290-301. https://doi.org/https://doi.org/10.1016/j.ibror.2020.10.006

Stickgold, R., James, L., \& Hobson, J. A. (2000). Visual discrimination learning requires sleep after training. Nature Neuroscience, 3(12), 12371238. https://doi.org/10.1038/81756

Tang, Y.-Y., Hölzel, B. K., \& Posner, M. I. (2015). The neuroscience of mindfulness meditation. Nature Reviews Neuroscience, 16(4), 213-225. https://doi.org/10.1038/nrn3916

Tomporowski, P. D. (2003). Effects of acute bouts of exercise on cognition. Acta Psychologica, 112(3), 297-324. https://doi.org/10.1016/s00016918(02)00134-8

Tunovic, S., Press, D. Z., \& Robertson, E. M. (2014). A Physiological Signal That Prevents Motor Skill Improvements during Consolidation. Journal of Neuroscience, 34(15), 5302-5310. https://doi.org/10.1523/jneurosci.3497-13.2014

Van Doorn, J., Van Den Bergh, D., Böhm, U., Dablander, F., Derks, K. Draws, T., . . . Wagenmakers, E.-J. (2020). The JASP guidelines for conducting and reporting a Bayesian analysis. Psychonomic Bulletin \& Review. https://doi.org/10.3758/s13423-020-01798-5

Walker, M. P. (2005). A refined model of sleep and the time course of memory formation. Behavioral and Brain Sciences, 28(1), 51-64. https://doi.org/10.1017/s0140525x05000026

Walker, M. P., Brakefield, T., Hobson, A. J., \& Stickgold, R. (2003). Dissociable stages of human memory consolidation and reconsolidation. Nature, 425(6958), 616-620. https://doi.org/10.1038/nature01930

Walker, M. P., Brakefield, T., Morgan, A., Hobson, J. A., \& Stickgold, R. (2002). Practice with Sleep Makes Perfect. Neuron, 35(1), 205-211. https://doi.org/10.1016/s0896-6273(02)00746-8

Walker, M. P., \& Stickgold, R. (2004). Sleep-Dependent Learning and Memory Consolidation. Neuron, 44(1), 121-133. https://doi.org/10.1016/j.neuron.2004.08.031

Wetzels, R., Matzke, D., Lee, M. D., Rouder, J. N., Iverson, G. J., \& Wagenmakers, E.-J. (2011). Statistical Evidence in Experimental Psychology. Perspectives on Psychological Science, 6(3), 291-298. https://doi.org/10.1177/1745691611406923

Willingham, D. B., \& Goedert-Eschmann, K. (1999). The Relation Between Implicit and Explicit Learning: Evidence for Parallel Development. Psychological Science, 10(6), 531-534. https://doi.org/10.1111/14679280.00201

Wulf, G., \& Prinz, W. (2001). Directing attention to movement effects enhances learning: A review. Psychonomic Bulletin \& Review, 8(4), 648660. https://doi.org/10.3758/bf03196201

Yanagisawa, H., Dan, I., Tsuzuki, D., Kato, M., Okamoto, M., Kyutoku, Y., \& Soya, H. (2010). Acute moderate exercise elicits increased dorsolateral prefrontal activation and improves cognitive performance with Stroop test. NeuroImage, 50(4), 1702-1710. https://doi.org/10.1016/j.neuroimage.2009.12.023

Zhong, Q.-Y., Gelaye, B., Sánchez, S. E., \& Williams, M. A. (2015). Psychometric Properties of the Pittsburgh Sleep Quality Index (PSQI) in a Cohort of Peruvian Pregnant Women. Journal of Clinical Sleep Medicine, 11(08), 869-877. https://doi.org/10.5664/jcsm.4936 